\title{
Clostridium butyricum relieve the visceral hypersensitivity in mice induced by Citrobacter rodentium infection with chronic stress
}

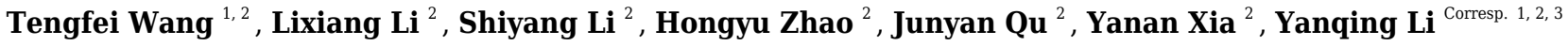 \\ ${ }^{1}$ Department of Gastroenterology, Qilu Hospital, Cheeloo College of Medicine, Shandong University, Jinan, Shandong, China \\ 2 Laboratory of Translational Gastroenterology, Qilu Hospital, Cheeloo College of Medicine, Shandong University, Jinan, Shandong Province, China \\ 3 Robot engineering laboratory for precise diagnosis and therapy of GI tumor, Qilu Hospital, Cheeloo College of Medicine, Shandong University, Jinan, \\ Shandong Province, China \\ Corresponding Author: Yanqing Li \\ Email address: liyanqing@sdu.edu.cn
}

Background: Visceral hypersensitivity is a common symptom in patients with post-infectious irritable bowel syndrome (PI-IBS), and change of the microbiota is a vital etiological factor of it. Clostridium butyricum (C.butyricum) is one of the probiotics which is reported as the active components in the treatment of IBS, especially IBS with diarrhea. Citrobacter rodentium (C. rodentium) is an enteropathogenic bacteria which can produce self-limiting colitis in mice, which have been used to produce a PI-IBS-like mice model. Whether C.butyricum could influence the visceral hypersensitivity and gut microbiota of PI-IBS is still unknown. Our study aimed to examine whether the intervention of $C$. butyricum or antibiotics could affect the etiology of visceral hypersensitivity.

Methods: C57BL/6 male mice were gavaged with the $C$. rodentium to induce a infective colitis. The C.butyricum and antibiotic compound were used to intervene the infected mice 3 days later. A 9-days chronic water avoidance stress (WAS) process was implemented to help induce the visceral hypersensitivity. The abdominal withdrawal reflex (AWR) score was assayed to indicate the visceral hypersensitivity of different groups. On the 7th, 14th, and 30th days after infection, mice feces were collected and high-throughput sequencing was carried out to analyze their gut microbiota.

Results: Combined post the $C$. rodentium infection plus chronic stress (WAS) could induce the visceral hypersensitivity in mice. Treatment of the $C$. butyricum after $C$. rodentium infection could relieve visceral hypersensitivity of mice, while no difference was observed in the antibiotic treatment group. The gut microbiota diversity of $C$. rodentium infected mice was similar to the no-infected mice, while there showed different microbial communities structure between them. The Shannon and Chao indexes significantly decreased in the antibiotic treatment group compared to other groups at 7th, 14th, and 30th post-infection days, while treatment of $C$. butyricum could maintain the indexes within normal range. At day 14 after infection, the structure of microbiota headed towards normality after the $C$. butyricum treatment. After the WAS, the Shannon and Chao indexes of the control group decreased and the structure of microbiota changed. The $C$. butyricum treatment could prevent these changes of the gut microbiota induced by WAS.

Conclusion: $C$. butyricum could relieve the visceral hypersensitivity in mice induced by $C$. rodentium infection plus chronic stress. It could also remodel the microbiota change caused by the infection and chronic stress. It may be a more effective treatment strategy for PI-IBS than antibiotics. 
1 Clostridium butyricum relieve the visceral

2 hypersensitivity in mice induced by Citrobacter

3 rodentium infection with chronic stress

4

5 Tengfei Wang ${ }^{12}$, Lixiang $\mathrm{Li}^{2}$, Shiyang $\mathrm{Li}^{2}$, Hongyu Zhao ${ }^{2}$, Junyan $\mathrm{Qu}^{2}$, Yanan $\mathrm{Xia}^{2}$, Yanqing $6 \mathrm{Li}^{1,2,3 *}$

7

81 Department of Gastroenterology, Qilu Hospital, Cheeloo College of Medicine, Shandong

9 University, Jinan, Shandong Province, China

102 Laboratory of Translational Gastroenterology, Qilu Hospital, Cheeloo College of Medicine,

11 Shandong University, Jinan, Shandong Province, China

123 Robot engineering laboratory for precise diagnosis and therapy of GI tumor, Qilu Hospital,

13 Cheeloo College of Medicine, Shandong University, Jinan, Shandong Province, China

14

*Corresponding Author:

20

Yanqing Li

21 No. 107, Wenhuaxi Road, Jinan, China

22 Fax\& Telephone: +86 53182166090

23 E-mail: liyanqing@sdu.edu.cn 


\section{Abstract}

26 Background: Visceral hypersensitivity is a common symptom in patients with post-infectious

27 irritable bowel syndrome (PI-IBS), and change of the microbiota is a vital etiological factor of it.

28 Clostridium butyricum (C.butyricum) is one of the probiotics which is reported as the active

29 components in the treatment of IBS, especially IBS with diarrhea. Citrobacter rodentium $(C$.

30 rodentium) is an enteropathogenic bacteria which can produce self-limiting colitis in mice, which

31 have been used to produce a PI-IBS-like mice model. Whether C.butyricum could influence the

32 visceral hypersensitivity and gut microbiota of PI-IBS is still unknown. Our study aimed to

33 examine whether the intervention of C. butyricum or antibiotics could affect the etiology of

34 visceral hypersensitivity.

35 Methods: C57BL/6 male mice were gavaged with the $C$. rodentium to induce a infective colitis.

36 The C.butyricum and antibiotic compound were used to intervene the infected mice 3 days later.

37 A 9-days chronic water avoidance stress (WAS) process was implemented to help induce the

38 visceral hypersensitivity. The abdominal withdrawal reflex (AWR) score was assayed to indicate

39 the visceral hypersensitivity of different groups. On the 7th, 14th, and 30th days after infection,

40 mice feces were collected and high-throughput sequencing was carried out to analyze their gut

41 microbiota.

42 Results: Combined post the $C$. rodentium infection plus chronic stress (WAS) could induce the

43 visceral hypersensitivity in mice. Treatment of the $C$. butyricum after $C$. rodentium infection

44 could relieve visceral hypersensitivity of mice, while no difference was observed in the antibiotic

45 treatment group. The gut microbiota diversity of $C$. rodentium infected mice was similar to the

46 no-infected mice, while there showed different microbial communities structure between them.

47 The Shannon and Chao indexes significantly decreased in the antibiotic treatment group 
48 compared to other groups at 7 th, 14 th, and 30th post-infection days, while treatment of $C$.

49 butyricum could maintain the indexes within normal range. At day 14 after infection, the

50 structure of microbiota headed towards normality after the C. butyricum treatment. After the

51 WAS, the Shannon and Chao indexes of the control group decreased and the structure of

52 microbiota changed. The C. butyricum treatment could prevent these changes of the gut

53 microbiota induced by WAS.

54 Conclusion: C. butyricum could relieve the visceral hypersensitivity in mice induced by $C$.

55 rodentium infection plus chronic stress. It could also remodel the microbiota change caused by

56 the infection and chronic stress. It may be a more effective treatment strategy for PI-IBS than

57 antibiotics. 


\section{Introduction}

60 Irritable bowel syndrome (IBS) is a common functional gastrointestinal disorder which is defined

61 by chronic abdominal pain or discomfort and altered bowel habits, without histopathologic

62 findings [1]. It has a obviously high morbidity (15-23\%) across the industrial world, causing a

63 reduced quality of life in patients and a considerable socioeconomic burden [2, 3]. Acute

64 infection in the gastrointestinal tract has six-fold risk factor to the development of IBS [4]. There

65 is a sub-type named post-infectious IBS (PI-IBS) characterized by new occurrence and frequent

66 abdominal pain that exhibits visceral hypersensitivity, with altered bowel habits (mostly

67 diarrhea), following the experience of an acute gastroenteritis episode [5]. PI-IBS represents

68 about $4 \%$ to $36 \%$ of all IBS cases [6]. Patients typically complain of the abdominal pain

69 symptoms which persist for years and the increasing of visceral hypersensitivity is commonly

70 found among them $[7,8]$.

71 Although the pathogenesis of PI-IBS is indistinct and complicated, there is plenty of evidences

72 showing that the intestinal barrier function dysbiosis [9], low grade inflammation of intestinal

73 mucosa $[10,11]$, neuroendocrine crosstalk [12], and the changes of the gastrointestinal

74 microbiota $[13,14,15]$ are related to it. Remarkably, gut microbial dysbiosis or inflammation

75 plays a key role in the pathogenesis of abdominal hypersensitivity of PI-IBS [16]. There are

76 increases in the numbers of Firmicutes and Proteobacteria, while decrease in numbers of

77 Bacteroidetes $[14,15]$. However, there is a relative paucity of research assessing the mechanism

78 between visceral hypersensitivity and the gut microbial remodeling of PI-IBS.

79 The guidelines of therapeutic strategy for PI-IBS are insufficient, there are still several clinical

80 therapies for the gastroenteritis episode, which can be divided into general treatment

81 (antidiarrheal and fluid supplement) and modifications of the gut microbiome (probiotics and 
82 antibiotics) [17]. The former treatment strategy has little influence on abdominal pain and 83 uncomfortable symptoms, and the treatment of various antibiotic drugs can induce dramatic and

84 irretrievable changes in the gut microbiota [18]. On the other hand, probiotics have been reported

85 to be the active component used in this field. With colonized into the intestinal tract and

86 remodeling of the gut microbiota in childhood gastroenteritis occasions [19], probiotics have

87 gained a great attention in recent years. The C. butyricum is a type of Gram-positive anaerobic

88 bacterium in gut microbiota, and it could regulate intestinal immune function and prevent colitis

89 in mice [20]. It was reported that C. butyricum can improves symptoms, quality of life and stool

90 frequency in IBS-D patients [21]. Other kind of probiotics such as Lactobacillus and

91 Bifidobacteria preperations also have potential in reducing abdominal symptoms and regulating

92 bowel habits of IBS-D [22]. Although there is phenomenal evidence for the C. butyricum

93 treatment on IBS, whether it could influence the visceral hypersensitivity and gut microbiota of

94 PI-IBS is is still unknown.

95 It is well known that Citrobacter rodentium, which resembles the pathogenic factor pathogens

96 enteropathogenic E. coli (EPEC) and enterohaemorrhagic E. coli (EHEC) in humans [23], can

97 produce a transient and self-limiting colitis in mice [24]. It is interesting that the infectious mice

98 treated with the chronic water avoidance test will have induced visceral hypersensitivity.

99 In this study, we prepared the protocol that we could use mice subjected to the $C$. rodentium

100 infection plus chronic water stress as the rodent model of PI-IBS. According to the above-

101 mentioned research results, our idea is that intervention of C. butyricum may actually involve in

102 or influence the etiology of visceral hypersensitivity in PI-IBS through microbiotia modification.

103 Materials \& Methods

104 Mice Model 
105 Thirty C57BL/6 male mice (5-6 weeks old, 15-20 g) were purchased from SPF Biotechnology

106 Co., Ltd. (Beijing, China), and twenty-four of them were randomly assigned to four groups: the

107 C. rodentium gavaged group (C.rodentium group), the C. rodentium + C. butyricum intervention 108 group (C. butyricum group, or probiotic group), the C.rodentium + antibiotics group (antibiotic 109 group) and the control group with PBS gavaged group ( $\mathrm{n}=6$ per group). The C.rodentium

110 infected groups were gavaged with C. rodentium $\left(2 \times 10^{9}\right.$ colony forming units $(\mathrm{CFU}), 200 \mu \mathrm{l}$ of 111 PBS) on day 0. Mice in the probiotic and antibiotic groups were gavaged with C. rodentium

112 together on day 0 , while C.butyricum or antibiotics were joined in 3 days later. Mice in the 113 probiotic group were gavaged C. butyricum $\left(1 \times 10^{9} \mathrm{CFU}, 200 \mu \mathrm{l}\right.$ of PBS) daily for one week.

114 Mice in the antibiotic group were given gentamycin (Sigma) at $20 \mathrm{mg} / \mathrm{kg} / \mathrm{d}$ and ampicillin 115 (Sigma) at 500mg/kg/d in drinking water for one week (Fig 1). The rest of the six mice were 116 given C.rodentium and deeply euthanizing with 5-minutes $\mathrm{CO} 2$ asphyxiation at 30\% Chamber

117 Replacement Rates at day $0(\mathrm{n}=2)$, day $5(\mathrm{n}=2)$ and day $21(\mathrm{n}=2)$ after infection, and their distal 118 colonal tissues were collected for HE Staining. Mice that survived the study were bred for our 119 other experiments. All mice were maintained on a 12-h light/dark cycle, with room temperature 120 of $22 \circ \mathrm{C}-24 \circ \mathrm{C}$ and relative humidity of $50-60 \%$. Mice were allowed aseptic food and water in a 121 level 2 animal feeding facility at Shandong University Laboratory Animal Center, and we have 122 mixed the bedding for all mouse cages randomly at the beginning of the mice experiment. All 123 procedures in this experiment were in accordance with the ARRIVE guidelines, and were 124 approved by the Shandong University Laboratory Animal Center and the Ethics Committee on 125 Animal Experiment of Shandong University Qilu Hospital (animal experiment proof certificate 126 number: Dull-2020-11).

127 Culture of $\boldsymbol{C}$. rodentium and $\boldsymbol{C}$. butyricum 
128 A single $C$. rodentium (ATCC51459) colony was inoculated into Luria broth (LB) medium and

129 grown overnight (at $37^{\circ} \mathrm{C}$ and $150 \mathrm{rpm}$ for $12 \mathrm{~h}$ ). The $C$. butyricum was seperated from $C$.

130 butyricum capsules (ATaiNing, Qingdao Eastsea Pharmaceutical Co., Ltd., China, 420 mg per

131 capsule, $\left.1.5 \times 10^{7} \mathrm{CFU} / \mathrm{g}, \mathrm{CGMCC} 0313.1\right)$ and cultured anaerobically at $37^{\circ} \mathrm{C}$ for $14 \mathrm{~h}$ in De

132 Man, Rogosa and Sharpe (MRS) medium.

133 Water-avoidance stress (WAS)

134 The $C$. rodentium infected mice group, the probiotic group, and the antibiotic group were all 135 given a 9-days chronic water avoidance stress (WAS) paradigm (days 21-30 after infection). For

1361 hour each day of the chronic stress period, mice were placed on a dry platform (70 $\mathrm{mm}$

137 diameter) in an acrylic bucket of water (280 $\mathrm{mm}$ diameter), standing $20 \mathrm{~mm}$ above water level

138 and $10 \mathrm{~mm}$ below the brim of the bucket. Mice were stressed within a single period of chronic

139 stress (9 days of WAS concluding on post-gavage day 21) as shown in Fig.1.

140 Behavioral Testing

141 Behavioral responses to CRD were assessed in four groups starting 1 days after the water-

142 avoidance stress by measuring the abdominal withdrawal reflex (AWR) using a semiquantitative

143 score. The testing of AWR indicated an express contraction in the abdominal wall musculature.

144 Distention water balloons (6-Fr, $2 \mathrm{~mm}$ external diameter) were placed in the descending colons

145 of mildly sedated mice (4\% isoflurane, temporary inhaled anesthesia) and secured by taping the

146 mouse's tail, and then they were put into a transparent acrylic box. The mice were then housed in

147 small boxes as a recovery room and allowed to wake up and adapt (1 hour). Measurement of the

148 AWR consisted of visual observation of the animal response to graded CRD $(0.1,0.15,0.2,0.25$

149 and $0.3 \mathrm{ml}$ of water injected into balloons) by double-blinded observers and assignment of the

150 AWR score. Each mouse was tested three times at each graded CRD and averaging for analysis. 


\section{HE Staining}

152 The HE staining was conducted in the colonic histology specimens of the six C.rodentium

153 infected mice. The paraffin-embedded distal colonic tissues were cut into $4 \mu \mathrm{m}$ longitudinal

154 sections, after proper specimen processing which involves dehydration, clearing, and paraffin

155 infiltration. After deparaffinization and rehydration, the sections were stained with hematoxylin

156 solution for 2 min followed by $3-4$ drips of $1 \%$ acid ethanol (1\% $\mathrm{HCl}$ in $70 \%$ ethanol) for 2 sec.

157 After the rinse by water, they were stained with eosin solution for 2-3 min and followed by

158 dehydration with graded alcohol and xylene. The slides were observed and photographed using a

159 fluorescence microscope (Nikon TI-FLC-E; Nikon, Tokyo, Japan).

$16016 \mathrm{~S}$ gene pyrosequencing analysis of mice fecal samples

161 At the point-in-time of orange triangles in Fig. 1, feces samples were collected at day 0 and in

162 each week after C. rodentium infection and they were weighed and homogenized in sterile PBS

163 for microbiota analysis. Fecal samples from the four group mice were collected and DNA was

164 extracted, and the 16S rRNA gene was amplified and sequenced. The 16S rRNA pyrosequencing

165 was processed at Majorbio (Shanghai, China) by using the Illumina Miseq system. The Chao

166 index, Sobs index and Shannon index were calculated to assess the alpha-diversity in each

167 sample. Cluster analysis based on the Euclidean distance was conducted based on the relative

168 abundances of all operational taxnomic units (OTUs). The principal co-ordinates analysis

169 (PCoA) based on the Bray-Curtis distance was done in order to performed to assess the beta-

170 diversity.

\section{Data analysis}

172 Data are expressed as mean $\pm \mathrm{SD}$, and error bars in figures represent SD. The statistical analysis

173 of AWR scores was performed using paired Student's t-tests, and significance was assigned 
174 when $* P<0.05, * * P<0.01, * * * P<0.001, \# \# P<0.01$. The raw data of $16 \mathrm{~S}$ gene pyrosequencing

175 were clustered into OTUs based on a 97\% similarity and analyzed through RDP classifier 2.11.

176 The community composition at each taxonomic level was calculated by Bayes algorithm. The

177 OTUs were then imported into Mothur v 1.38.1 for analyzing the rarefaction curves and alptha

178 diversity. The specific taxa were identified by the Linear discriminant analysis Effect Size

179 (LEfSe) analysis with the value of Kruskal -Wallis sum-rank test set to 0.05. The taxonomy of

180 each 16S rRNA gene sequence was analyzed by RDP Classifier algorithm

181 (http://rdp.cme.msu.edu/) against the Silva (SSU132) 16S rRNA database using confidence

182 threshold of 70\%. SPSS was used to analyze the data, a 95\% confidence level, $P<0.05$ was used.

183

184 Results

185 1. $C$. rodentium infection plus repeated water-avoid stress caused the visceral

186 hypersensitivity in mice

187 All mice exposed to $C$. rodentium alone showed signs of colitis early in the infection (Day 1 to

188 Day 4), such as soft stools, rough body hair, languid movement and temporary body weight loss

189 (Day 2 to Day 5). Two-week post infection, these symptoms gradually faded away, and all mice

190 finally recovered from infectious colitis and brought into next experiments. On the $30^{\text {th }}$ days after

191 C. rodentium infection and WAS treatment, the AWR score showed that the infectious group had

192 a lower pain tolerance for colorectal distension compared with the control group $(\mathrm{n}=6, P<0.01)$

193 as shown in Fig.2A. Also the colonic inflammation pathological changes of the infectious group

194 had recovered to normal within the assessment of the histopathological score (Fig.2B). Based on

195 these phenomena, we can draw the conclusion that $C$. rodentium infection and AWR stress could

196 produce a PI-IBS-like mice model.

Peer) reviewing PDF | (2021:01:57555:1:2:NEW 7 May 2021) 
197 2. C. butyricum could relieve the visceral hypersensitivity of PI-IBS-like mice.

198 We evaluated whether the visceral hypersensitivity caused by $C$. rodentium infection and WAS 199 could be influenced with probiotics or antibiotics treatment. It was observed that mice treated 200 with the C. butyricum presented lower AWR scores in the behavioral testing than C. rodentium 201 infected mice group $(\mathrm{n}=6, P<0.05)$. However, the gentamycin-ampicillin treatment group did not 202 show this characteristic $(\mathrm{n}=6, P>0.05)$ (Fig. 2A).

203 3. The OTUs of gut microbes tremendously decreased after antibiotics treatment, which 204 was not observed in $C$. butyricum treatment mice.

205 The 16S rRNA sequence was performed to investigate the change of the gut microbiota. It was 206 found that the number of OTUs were among $661-673$ at the beginning in different groups. The 207 C. rodentium infection and C. butyricum treatment did not affect the number of OTUs. However, 208 the number of OTUs obviously decreased to 580 at day 7 and further tremendously decrease to 209122 at day 14 in antibiotic treatment group (Fig. 3B). After WAS, the number of OTUs in 210 antibiotic group decreased to 111 at day 30 (Fig. 3B). At day 30, there were only 69 OTUs which 211 were shared in the four groups. In contrast, there were 456 OTUs which were shared in the three 212 groups except for the antibiotics group (Fig.3A). These results demonstrated the antibiotic indeed 213 destroy the abundance of microbiota in PI-IBS-like mice, while C. butyricum did not influence it. 214 4. The $\alpha$-diversity of gut microbiota obviously changed after antibiotics treatment, and the 215 C. butyricum could maintain them within normal range.

216 The $\alpha$-diversity of gut microbiota was analyzed and the results are shown in Fig. 4. It was found 217 that there was no difference in the Shannon and Chao indexes at day 0 (data not shown). After $C$. 218 rodentium infection, the Shannon and Chao indexes were not affected at day 7, day 14 and day 219 30. The treatment of C. butyricum also did not affect the Shannon and Chao indexes. However, 
220 the Chao index of the antibiotics group significantly decreased compared with the control group

221 (519.45 \pm 52.71 VS. $436.72 \pm 36.68, P<0.05)$ at day 7. Also, both the Shannon and Chao indexes of

222 the antibiotics group were significantly lower than those of the C. butyricum-treatment group

223 (3.45 $\pm 0.41 \mathrm{VS} 4.26 \pm 0.41, P<0.01 ; 436.72 \pm 36.68 \mathrm{VS} .524 .12 \pm 91.84, P<0.05)$. At day 14 , the

224 Shannon and Chao indexes of the antibiotics group decreased and were significantly lower than

225 those of the other three groups $(P<0.001)$. At day 30, the Shannon and Chao indexes of the

226 antibiotics group were still significantly lower than those of the C. rodentium infection group and

227 the C. butyricum-treatment group $(P<0.001)$.

228 5. The $\beta$-diversity of the gut microbiota changed in different groups.

229 The $\beta$-diversity of the gut microbiota was analyzed based on PCoA and the results are shown in

230 Fig 5 . The structure of the gut microbiota was nearly the same at day $0(\mathrm{R}=0.01, P=0.41)$. It was

231 found that $C$. rodentium infection did not affect the structure of the gut microbiota compared

232 with the control group $(\mathrm{R}=0.16, P=0.09)$. However, antibiotic treatment significantly affected the

233 structure of the gut microbiota at day $7(\mathrm{R}=0.32, P=0.001)$ and day $14(\mathrm{R}=0.55, P=0.001)$. At

234 day 14, the structure of the microbiota was similar between the control and the C. butyricum

235 groups. However, there were significant differences between the four groups $(P<0.05)$ (Fig S1).

236 After the WAS, the difference between the antibiotic treated group and the other three groups

237 was further increased $(\mathrm{R}=0.58, P=0.001)$ (Fig S2).

238 6. The microbiota composition changed in different groups.

239 The main genera of the gut micriobiota (the percentages were above 1\%) included more than 38

240 genera in all groups including Muribaculaceae, Lactobacillus, Bacteroides, Lachnospiraceae,

241 Ileibacterium, Alistipes, and Helicobacter, ect. (Fig. 6A). The specific taxa that most likely

242 contributed to the differences between the four groups at day 30 were determined by linear 
243 discriminant analysis effect size (Fig. 6B). The genera Lactobacillus, Turicibacter and

244 Flavobacteriaceae were enriched in the control group while the genera Muribaculaceae,

245 Lachnospiraceae, Helicobacter, Ileibacterium, Alistipes, and Ruminococcus were enriched in the

246 C. rodentium infection group. After treatment with antibiotics, the genera Bacteroides, Blautia,

247 Parasutterella, Parabacteroides, and Akkermansia were enriched. In contrast, the genera

248 Oscillospirales, Mucispirillum, Dubosiella, and Odoribacter were enriched in the C. butyricum-

249 treatment group. The amount of genera Lactobacillus and Odoribacter in the four groups are

250 shown in Fig. 6C and 6D. It was found that the amount of the genus Lactobacillus in the control

251 group was higher than those of other groups $(P<0.01)$. It increased in $C$. butyricum-treatment

252 group without significant difference. Furthermore, the genus Odoribacter in the C. butyricum-

253 treated group was higher than those of the other three groups $(P<0.05)$.

\section{Discussion}

255 In this study, after treatment with C.butyricum, the PI-IBS mouse model presented a lower AWR 256 scores which means mitigatory peritoneal hypersensitivity. In contrast, antibiotics treatment had 257 no effects on hypersensitivity. We also found that C.butyricum treatment could remodeling of the 258 gut microbiota. However, antibiotics treatment could induce a drastic change in the intestinal 259 microbiota.

260 In recent years, it was reported that lots of probiotic supplements have favorable effects on 261 IBS as revealed through in clinical cases and animal studies [25,26]. As the most commonly used 262 probiotics, Bifidobacterium is of great benefit to on abdominal pain and bloating in IBS patients

263 [21]. Lactobacillus acidophilus improve an improvement of barrier function and reduce

264 cytokines secretion, which contribute to resulting in the benefit of relieving visceral

265 hypersensitivity of IBS [27]. There was also a PI-IBS rodent model study to induce colitis 
266 through the Trichinella, and the intervention of Lactobacillus and yeast showed a decrease of 267 visceral hypersensitivity in mice [28]. The Trichinella causes persistent and histoclastie infection 268 in gut tissue [29], which may not reflect the pathogenic condition of PI-IBS. In this study, the 269 infection of $C$. rodentium is a self-limited colitis in mice which have a histological recovery in 27010 days after infection [30]. This rodent model could mimic a similar pathological and

271 immunological state of PI-IBS cases. The C.butyricum is a well-known probiotic which was used 272 for therapeutic strategy for IBS patients [21,31]. It revealed an important role in a 273 hypersensitivity-relieving effect in the C. rodentium infection plus WAS mice model (Fig. 2A).

274 Antibiotics are commonly used in the treatment of infectious diarrhea. In this study, an antibiotic 275 mixture of gentamycin-ampicillin was also used to treat the $C$. rodentium infection. Though the 276 fecal character greatly changed, it demonstrated no significant differences in hypersensitivity of 277 infected mice plus WAS (Fig. 2A). Thus, the C.butyricum might be a better choice for infectious 278 diarrhea than antibiotics.

279 There have been many studies on the pathogenesis of IBS, and one of the most important 280 explains is gut microbiota remodeling. Gastroenteritis episodes induce variation in the gut 281 microbiota, which usually return to normal in healthy people after recovery. However, those 282 developing PI-IBS may have a relative inability to restore their microbiota [32]. It was reported 283 that the C57BL/6 mice used in our study is one of C. rodentium-resistant mouse line which could 284 only get a self-limiting colitis and more mild symptoms of diarrhea and weight loss compare to 285 the sensitive mouse lines [33].The amount of C.rodentium were only about $10^{2}-10^{4} / \mathrm{g}$ feces 3 286 days after infection in the resistant mice [34]. And the $\alpha$-diversity remained stable and non287 significant decrease during the infection until day 14 [35]. However, the intervention of 288 C.rodentium did affect the structure of gut microbiota same as the published reports [34,36]. In 
289 this study, the structure of the microbiota was still not restored at day 14 after infection without 290 treatment or treatment with antibiotic (Fig. 5 and Fig. S1). In contrast, the structure of the 291 microbiota of the mice in the C.butyricum treatment group was similar to those of the control 292 group (Fig. S1), which indicated its effect on maintaining the balance of gut microbiota. Also, 293 chronic persistent stress, such as WAS, plays an important role in remodeling the gut microbiota 294 of mice [37].

295 In this study, we found that WAS treatment remodeled the microbiota and the Shannon and 296 Chao indexes even decreased in control group (Fig. 3). However, the Shannon and Chao indexes 297 did not change after WAS treatment in the C.butyricum treatment group. It was interesting that 298 only the structure of the microbiota of the C.butyricum treatment group did not change after 299 WAS (Fig. S2). This suggested that C.butyricum could protect the microbiota from chronic 300 persistent stress. Furthermore, the genera Odoribacter and Lactobacillus increased more in the 301 C.butyricum treatment group at day 30 than in the C. rodentium infections group and in the 302 antibiotic group. It was reported that higher baseline proportions of Odoribacter were related to 303 beneficial inflammatory-marker changes [38]. In addition, probiotics of the genus Lactobacillus 304 are also have potentially reduced abdominal symptoms of IBS $[39,40]$. These may be the reason why C. butyricum alleviates visceral hypersensitivity.

306 On the other hand, the antibiotics can affect the gut microbiota of PI-IBS [41]. In this study, 307 we found that the Shannon and Chao indexes as well as the structure of the microbiota 308 significantly changed after the treatment of antibiotics (Fig.4 and Fig.5) and the tendencies could 309 last for 30 days. It was also found that the genus Bacteroides increased at day 14 and 30 (Fig. 310 6A) in antibiotic group. The increase of the Bacteroidetes phylum composition may increase the 
311 susceptibility to infection and it is also abundant in PI-IBS patients [42]. Therefore, probiotics

312 are more suitable than antibiotics in remodeling the microbiota of patients after infection.

313 There may be some possible limitations in this study. First, whether or not probiotics include

314 other strains of Clostridium have the same effect on the visceral hypersensitivity induced by the

315 intestinal infection was not confirmed. Second, the PI-IBS-like rodent model can not identically

316 imitate the pathological conditions of PI-IBS patients, it need to continually improve. Thirdly,

317 we observed the $C$. butyricum could relive the visceral hypersensitivity and the gut microbiota

318 was closely related to it, the specific molecular or immune mechanism was not explored and

319 need further research.

320 Conclusions

321 In conclusion, it was found that the C. butyricum could relieve the visceral hypersensitivity in

322 mice induced by $C$. rodentium infection plus chronic stress. It was also superior to antibiotics in 323 remodeling the microbiota of the visceral hypersensitivity model mice. Thus, C. butyricum may 324 be a candidate for the treatment of PI-IBS.

325

326 Additional Information and Declarations

327 Funding

328 This study was supported by the National Natural Science Foundation of China (81873550), Key

329 Research and Development Program of Shandong Province (2019GHZ022). This study is also

330 supported by the Taishan Scholars Program of Shandong Province and National Clinical

331 Research Center for Digestive Diseases supporting technology project (2015BAI13B07).

\section{Competing Interests}

333 The authors declare there are no competing interests. 


\section{Author Contributions}

335 T. -F.W., L.-X.L.and Y.-Q.L. designed the experiments; T.-F.W. performed the experiments.T.336 F.W. and L.-X.L. analyzed the data, prepared the figures. S.-Y.L. provided the strain of 337 Citrobacter rodentium. T.-F.W. and L.-X.L. wrote manuscript; Y.-Q.L. obtained funding; Y.338 Q.L.and S.-Y.L. revised of the manuscript and all authors read the manuscript and approved the 339 final draft that was submitted. Thanks to Dr. Edward C. Mignot, Shandong University, for 340 linguistic advice.

\section{Animal Ethics}

342 The following information was supplied relating to ethical approvals: All procedures in this 343 experiment were in accordance with the ARRIVE guidelines, and were approved by the

344 Shandong University Laboratory Animal Center and the Ethics Committee on Animal 345 Experiment of Shandong University Qilu Hospital (animal experiment proof certificate number:

346 Dull-2020-11).

\section{Data Availability}

348 The 16S gene pyrosequencing data is available at NCBI BioProject database, BioProject ID:

349 PRJNA698375.The raw measurements are available in

350 https://www.ncbi.nlm.nih.gov/sra/PRJNA698375.

351 The sample name and group information were shown in Table S1.

\section{References}

$3531 \square$ Longstreth, G.F., et al., Functional bowel disorders. Gastroenterology, 2006. 130(5): p.1480-91. 
$3552 \square$ Guglielmetti, S., et al., Randomised clinical trial: Bifidobacterium bifidum MIMBb75

356 significantly alleviates irritable bowel syndrome and improves quality of life--a double-

357 blind, placebo-controlled study. Aliment Pharmacol Ther, 2011. 33(10): p. 1123-32.

$3583 \square$ Saha, L., Irritable bowel syndrome: pathogenesis, diagnosis, treatment, and evidence-

359 based medicine. World J Gastroenterol, 2014. 20(22): p. 6759-73.

$3604 \square$ Halvorson, H.A., C.D. Schlett, and M.S. Riddle, Postinfectious Irritable Bowel

361 Syndrome-A Meta-Analysis. The American Journal of Gastroenterology, 2006. 101(8): p.

$362 \quad 1894-1899$.

$3635 \square$ Barbara, G., et al., Rome Foundation Working Team Report on Post-Infection Irritable 364 Bowel Syndrome. Gastroenterology, 2019. 156(1): p. 46-58.e7.

$3656 \square$ Spiller, R. and E. Campbell, Post-infectious irritable bowel syndrome. Curr Opin 366 Gastroenterol, 2006. 22(1): p. 13-7.

$3677 \square$ Marshall JK, et al. Walkerton Health Study 604 Investigators. Eight year prognosis of 368 postinfectious irritable bowel syndrome following 605 waterborne bacterial dysentery.

369 Gut, 2010. 59: 605-611.

$3708 \square$ Lee, Y.Y., C. Annamalai, and S.S.C. Rao, Post-Infectious Irritable Bowel Syndrome.

371 Current Gastroenterology Reports, 2017. 19(11).

$3729 \square$ Cotton JA, Beatty JK, Buret AG. Host parasite interactions and pathophysiology in

373 Giardia infections.Int J Parasitol. 2011; 41(9):925-33.

$37410 \square$ Spiller RC, et al. Increased rectal mucosal enteroendocrine cells, T lymphocytes, and 375 increased gut permeability following acute Campylobacter enteritis and in post-

376 dysenteric irritable bowel syndrome. Gut. 2000; 47(6):804-11. 
$377 \quad 11 \square$ Barbara G, et al. Activated mast cells in proximity to colonic nerves correlate with 378 abdominal pain in irritable bowel syndrome. Gastroenterology. 2004;126:693-702.

$37912 \square \mathrm{Ng}$, Q.X., et al., The role of inflammation in irritable bowel syndrome (IBS). J 380 Inflamm Res, 2018. 11: p. 345-349.

$38113 \square$ Lupp C, et al. Host-mediated inflammation disrupts the intestinal microbiota and 382 promotes the overgrowth of Enterobacteriaceae. Cell Host Microbe. 2007;2:119-29.

$38314 \square$ Krogius-Kurikka L, Lyra A,et al. Microbial community analysis reveals high level 384 phylogenetic alterations in the overall gastrointestinal microbiota of diarrhoea385 predominant irritable bowel syndrome sufferers. BMC Gastroenterol. 2009;9:95.

$38615 \square$ Malinen E, Rinttilä T, Kajander K,et al. Analysis of the fecal microbiota of irritable 387 bowel syndrome patients and healthy controls with real-time PCR. Am J Gastroenterol. $388 \quad 2005 ; 100: 373-82$.

$38916 \square$ Su, T., et al., Altered Intestinal Microbiota with Increased Abundance of Prevotella 390 Is Associated with High Risk of Diarrhea-Predominant Irritable Bowel Syndrome.

391 Gastroenterol Res Pract, 2018. 2018: p. 6961783.

392 17 $\square$ Chey, W.D., J. Kurlander, and S. Eswaran, Irritable Bowel Syndrome. Jama, 2015. 393 313(9): p. 949.

394 18 $\square$ Rodino-Janeiro, B.K., et al., A Review of Microbiota and Irritable Bowel Syndrome: 395 Future in Therapies. Adv Ther, 2018. 35(3): p. 289-310.

396 19 $\square$ Freedman, S.B., et al., Multicenter Trial of a Combination Probiotic for Children 397 with Gastroenteritis. New England Journal of Medicine, 2018. 379(21): p. 2015-2026. 
$398 \quad 20 \square$ Hayashi A, et al. Kanai T. A single strain of Clostridium butyricum induces intestinal 399 IL-10-producing macrophages to suppress acute experimental colitis in mice. Cell Host 400 Microbe 2013; 13: 711-722.

$40121 \square$ Sun, Y.-Y., et al., The effect of Clostridium butyricum on symptoms and fecal 402 microbiota in diarrhea-dominant irritable bowel syndrome: a randomized, double-blind, 403 placebo-controlled trial. Scientific Reports, 2018. 8(1).

$40422 \square$ Koval, M., et al., Are There Any Different Effects of Bifidobacterium, Lactobacillus 405 and Streptococcus on Intestinal Sensation, Barrier Function and Intestinal Immunity in 406 PI- IBS Mouse Model? PLoS ONE, 2014. 9(3): p. e90153.

$40723 \square$ Mullineaux-Sanders, C., et al., Citrobacter rodentium-host-microbiota interactions: 408 immunity, bioenergetics and metabolism. Nat Rev Microbiol, 2019. 17(11): p. 701-715. $40924 \square$ Ibeakanma, C., et al., Citrobacter rodentium colitis evokes post-infectious 410 hyperexcitability of mouse nociceptive colonic dorsal root ganglion neurons. J Physiol, 411 2009. 587(Pt 14): p. 3505-21.

$41225 \square$ Ford, A.C., et al. Efficacy of prebiotics, probiotics, and synbiotics in irritable bowel 413 syndrome and chronic idiopathic constipation: systematic review and meta-analysis.

414 AmJ Gastroenterol, 2014. 109(10): p. 1547-61; quiz 1546, 1562.

$41526 \square$ Didari, T., et al., Effectiveness of probiotics in irritable bowel syndrome: Updated 416 systematic review with meta-analysis. World J Gastroenterol, 2015. 21(10): p. 3072-84. $41727 \square$ Zeng, J., et al., Clinical trial: effect of active lactic acid bacteria on mucosal barrier 418 function in patients with diarrhoea-predominant irritable bowel syndrome. Alimentary 419 Pharmacology \& Therapeutics, 2008. 28(8): p. 994-1002. 
$42028 \square$ Hong, K.B., et al., Effects of probiotic supplementation on post-infectious irritable 421 bowel syndrome in rodent model. BMC Complement Altern Med, 2019. 19(1): p. 195. $42229 \square$ Luo, X.-C., et al., Infection by the parasitic helminthTrichinella spiralisactivates a Tas $2 r$ 423 mediated signaling pathway in intestinal tuft cells. Proceedings of the National Academy of 424 Sciences, 2019. 116(12): p. 5564-5569.

$42530 \square$ Collins, J.W., et al. Citrobacter rodentium: infection, inflammation and the microbiota. Nat 426 Rev Microbiol, 2014. 12(9): p. 612-23.

$42731 \square$ Zhao, Q., et al. Clostridium butyricum alleviates intestinal low-grade inflammation in TNBS428 induced irritable bowel syndrome in mice by regulating functional status of lamina propria 429 dendritic cells. World J Gastroenterol, 2019. 25(36): p. 5469-5482.

$43032 \square$ Neal, K.R., L. Barker, and R.C. Spiller, Prognosis in post-infective irritable bowel 431 syndrome: a six year follow up study. Gut, 2002. 51(3): p. 410-3.

$43233 \square$ Vallance, B.A., et al., Host susceptibility to the attaching and effacing bacterial pathogen 433 Citrobacter rodentium. Infect Immun, 2003. 71(6): p. 3443-53.

$43434 \square$ Osbelt, L., et al., Variations in microbiota composition of laboratory mice influence 435 Citrobacter rodentium infection via variable short-chain fatty acid production. PLoS Pathog, $436 \quad 2020.16(3):$ p. e134.008448.

$43735 \square$ Cannon, T., et al., Characterization of the intestinal microbiota during Citrobacter 438 rodentium infection in a mouse model of infection-triggered Parkinson's disease. Gut Microbes, 439 2020. 12(1): p. 1-11.

$44036 \square$ Hoffmann, C., et al., Community-wide response of the gut microbiota to enteropathogenic 441 Citrobacter rodentium infection revealed by deep sequencing. Infect Immun, 2009. 77(10): p. $442 \quad 4668-78$. 
443 37 $\square$ Han, Y., et al., Hydrogen sulfide-mediated resistance against water avoidance stress-

444 induced gastritis by maintenance of gastric microbial homeostasis. Microbiologyopen, 2020.

445 9(1): p. e00951.

$44638 \square$ Hod, K., et al., The effect of a multispecies probiotic on microbiota composition in a clinical

447 trial of patients with diarrhea-predominant irritable bowel syndrome. Neurogastroenterol Motil,

$448 \quad 2018.30(12):$ p. e13456.

$44939 \square$ Bonfrate, L., et al., Effects of Bifidobacterium longum BB536 and Lactobacillus rhamnosus

450 HN001 in IBS patients. Eur J Clin Invest, 2020. 50(3): p. e13201.

$45140 \square$ Preston, K., et al., Lactobacillus acidophilus CL1285, Lactobacillus casei LBC80R and

452 Lactobacillus rhamnosus CLR2 improve quality-of-life and IBS symptoms: a double-blind,

453 randomised, placebo-controlled study. Benef Microbes, 2018. 9(5): p. 697-706.

$45441 \square$ Becattini, S., Y. Taur, and E.G. Pamer, Antibiotic-Induced Changes in the Intestinal

455 Microbiota and Disease. Trends in Molecular Medicine, 2016. 22(6): p. 458-478.

$45642 \square$ Downs, I.A., et al., Postinfection Irritable Bowel Syndrome. Journal of Clinical

457 Gastroenterology, 2017. 51(10): p. 869-877. 


\section{Figure 1}

Timeline for C.rodentium infection and C.butyricum/antibiotics intervention for mice.

C.rodentium gavage began at 0 days to $5-6$ weeks old mice except the control group. The $C$. rodentium $+C$. butyricum intervention group and the C.rodentium + antibiotics group were given the probiotics or antibiotics, reseperately. And the intervention last for 1 week (orange stripes). Three C.rodentium infectious groups were followed by water avoidance stress (WAS) for a period of 9 days (blue stripes). Mice feces samples were taken and stored weekly (yellow triangles) until day 30.

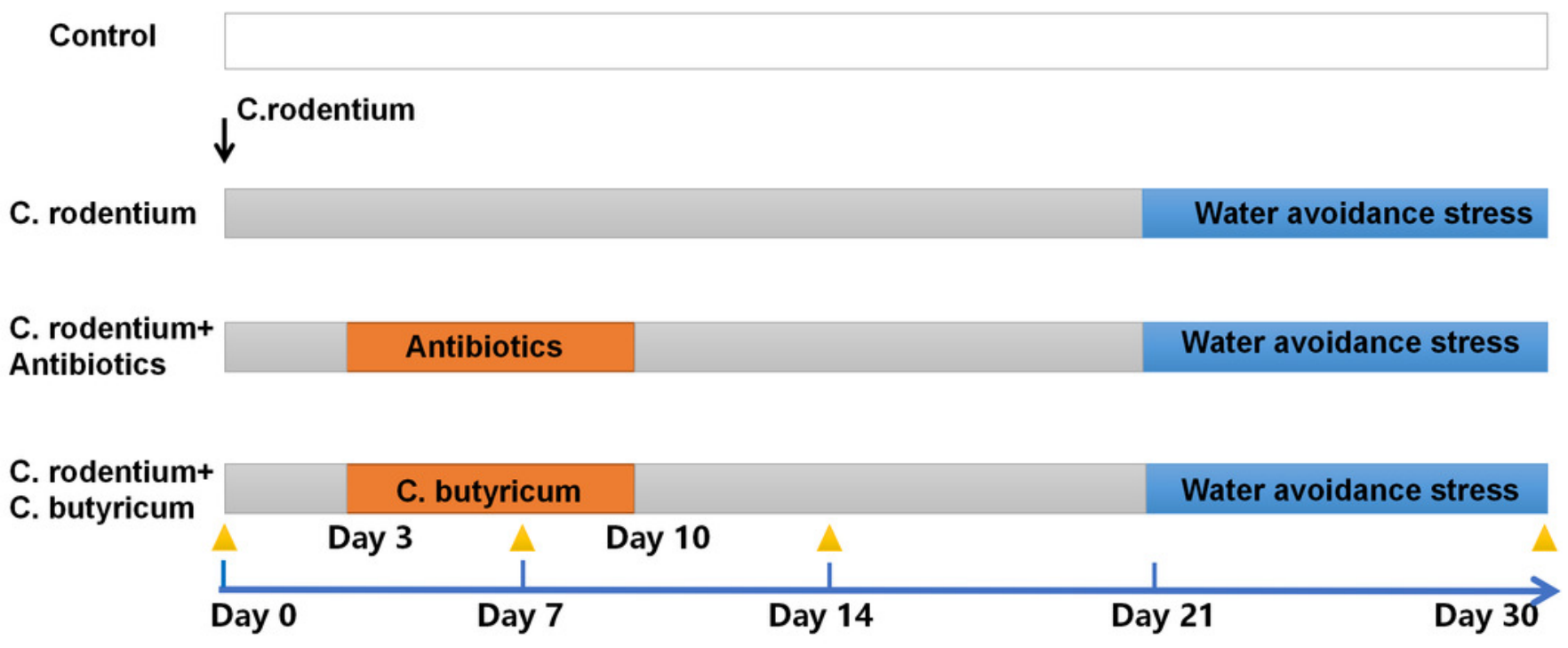




\section{Figure 2}

The AWR score and H\&E-stained distal colon sections of mice.

(A)Summary data ( $\mathrm{n}=6$ mice per group) illustrating the AWR scores which were the responses to graded CRD with water injection in mice subjected to the PBS-only control group (blue circles), C.rodentium-only (orange squares), C.butyricum co-treatment with C.rodentium (pink triangles), and gentamicin-ampicillin co-treatment with $C$. rodentium (green triangles).The AWR scores were higher in the WAS plus C.rodentium infectious group compare with control group ( $* * P<0.01)$, and the co-treatment of $C$. butyricum could reverse this tendency $(* P<0.05)$ while co-treatment of antibiotics had no such effect on it $(P>0.05)$. When the water injected into balloons was $0.15 \mathrm{ml}$, the AWR scores of C.rodentium group was apparently higher than control group ( $\# \# P<0.01$ ). Data are reported as mean $\pm \mathrm{SD}$; paird Student T-test. (B-D) H\&E-stained distal colon sections from C.rodentium infectious mice at 0 day (B), 5 days (C) and 21 days (D) after infection. No inflammation is evident in sections of untreated mice and 21 days after infection mice,while there was inflammatory cell infiltration and hyperplasia in the mucosa of $C$.rodentium infectious mice at day 5 . 


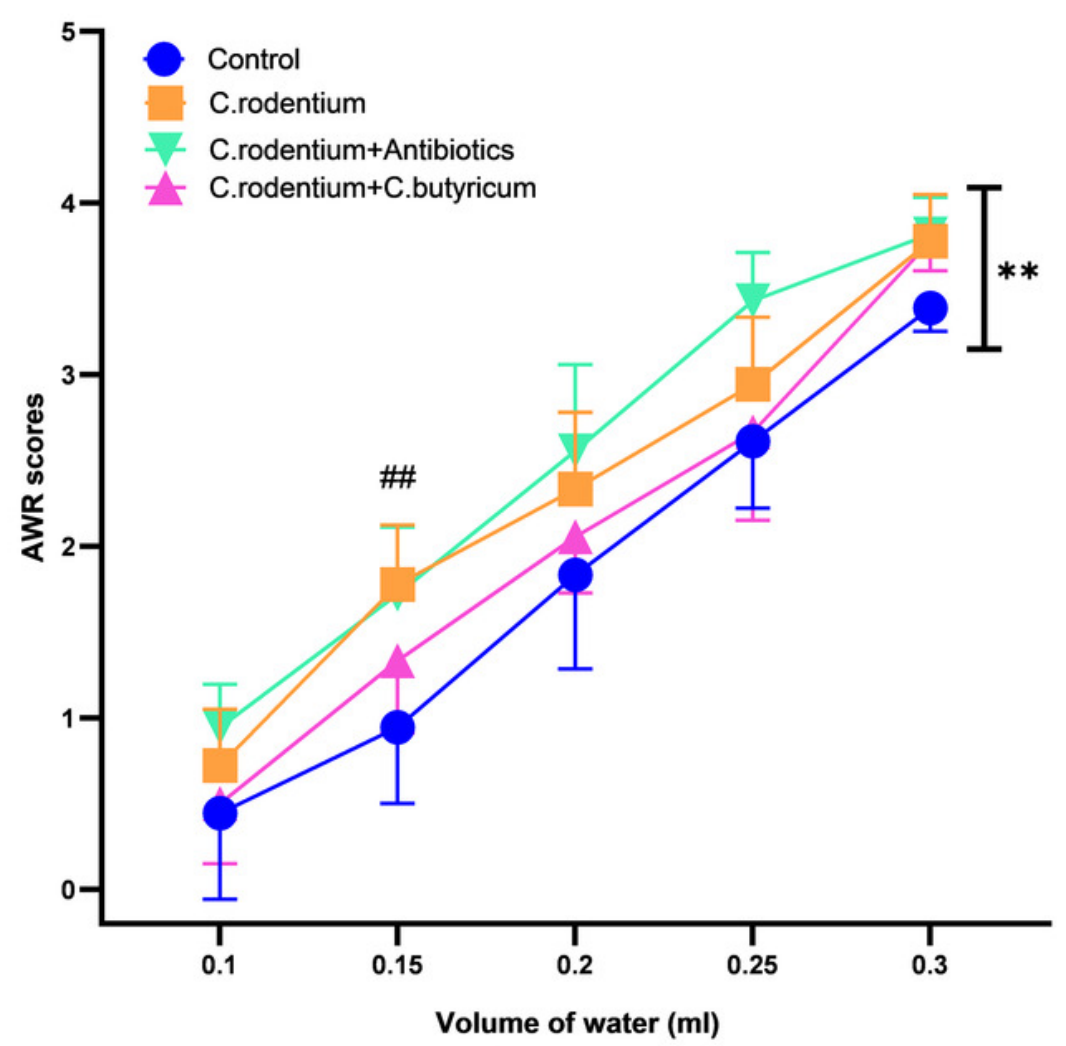

A

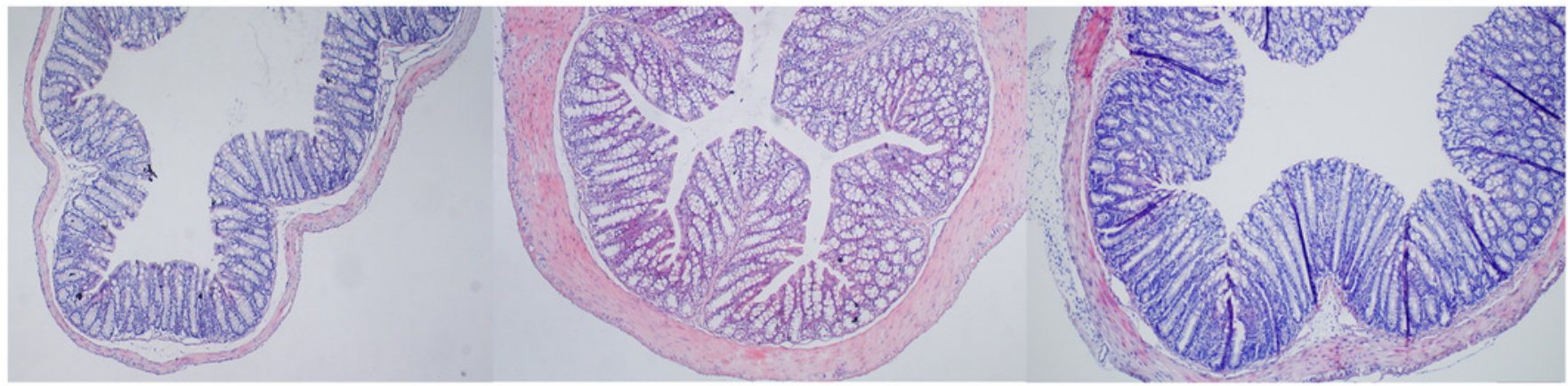

B Day $0,100 x$

C Day 5 after C.rodentium infection, $100 \mathrm{X}$

D D21 after C.rodentium infection, 100X 


\section{Figure 3}

Comparison of the OTU of the microbiota in different groups.

(A)The 16S rRNA sequence for the number of OTUs plotted to the Venn diagram of the four experimental groups at day 30 after WAS. There were only 69 OTUs which were shared in the four groups. In contrast, there were 456 OTUs which were shared in the three groups except for the antibiotics group. (B) OTU numbers were counted in the four groups at different times (day 0 , day 7 , day 14 and day 30 ). The antibiotic treatment after C.rodentium infection demonstrated a low bacterial species diversity compared with control and other infectious groups. 
A Venn (Day 30)
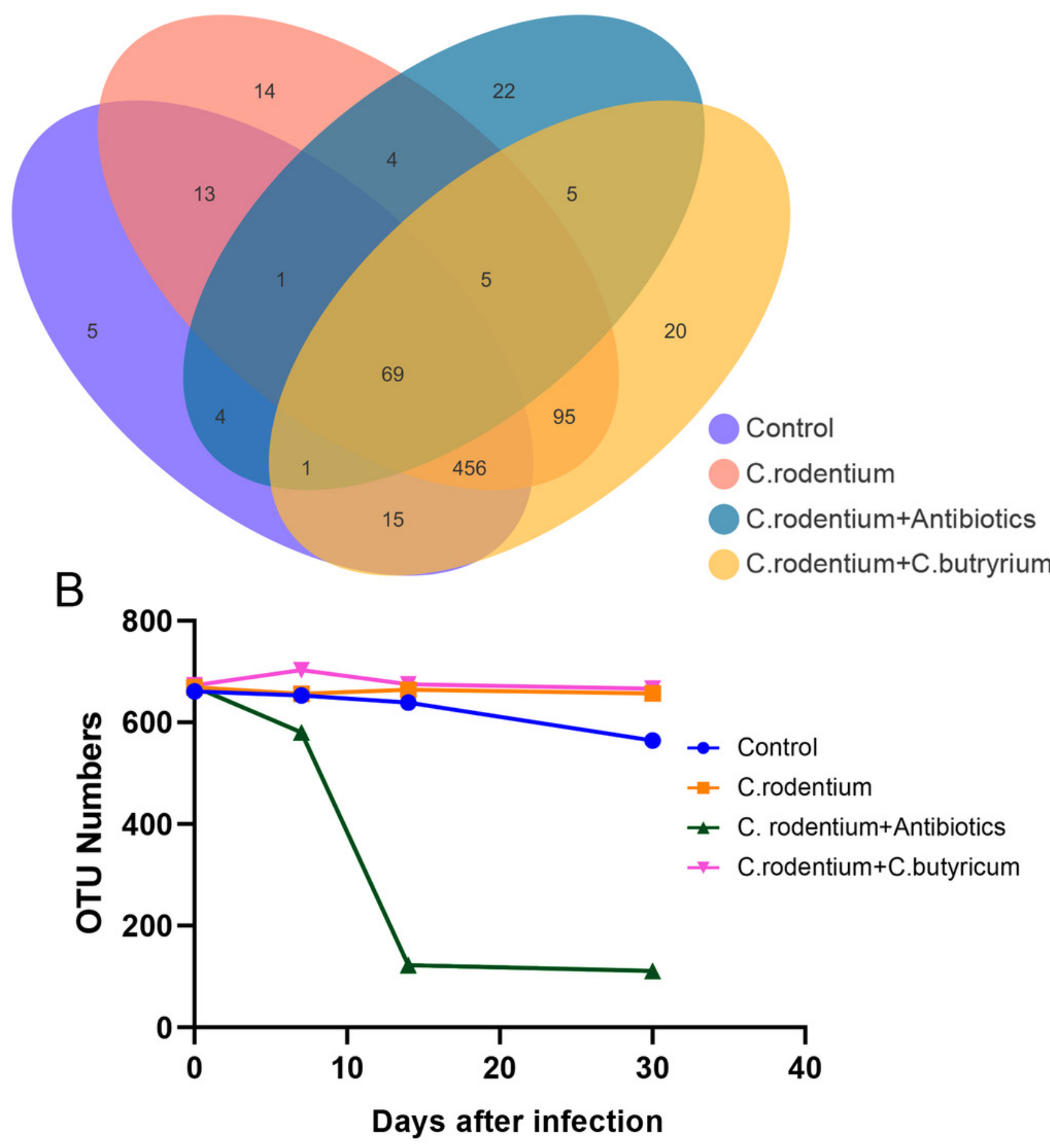
Figure 4

The $\alpha$-diversity of gut microbiota in the four groups at different times.

The Chao index of the antibiotic group decreased compared with the control group

$(519.45 \pm 52.71$ VS. $436.72 \pm 36.68, P<0.05)$ at day 7 ; both the Shannon and Chao indexes of the antibiotic group were also lower than the $C$. butyricum-treatment group $(3.45 \pm 0.41$ VS4.26 $\pm 0.41, P<0.01 ; 436.72 \pm 36.68 \mathrm{VS} .524 .12 \pm 91.84, P<0.05)$ at day 14 ; tay 30 , the Shannon and Chao indexes of the antibiotic group were still lower in antibiotic group compared with the other three groups $(P<0.001)$. The $C$. butyricum-treatment group did not affect the Shannon and Chao indexes at day 7, day 14 and day 30 .
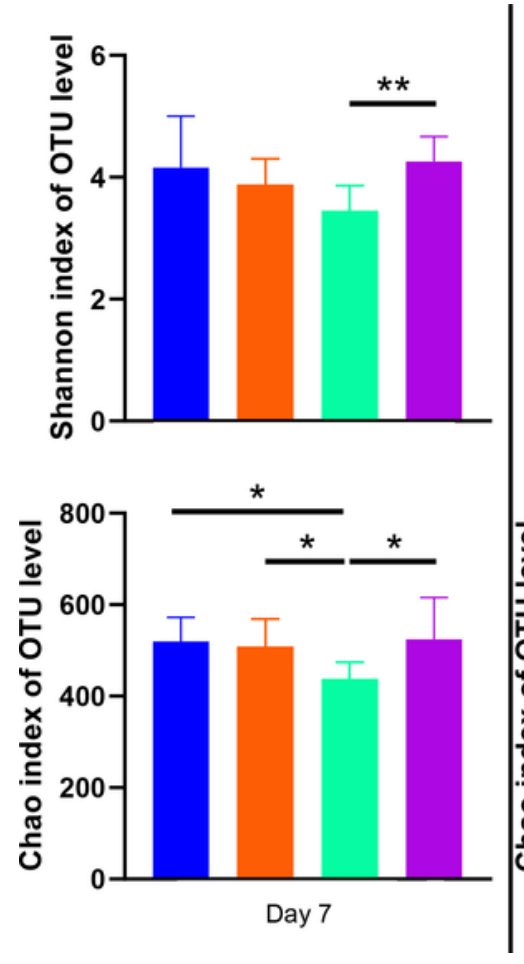
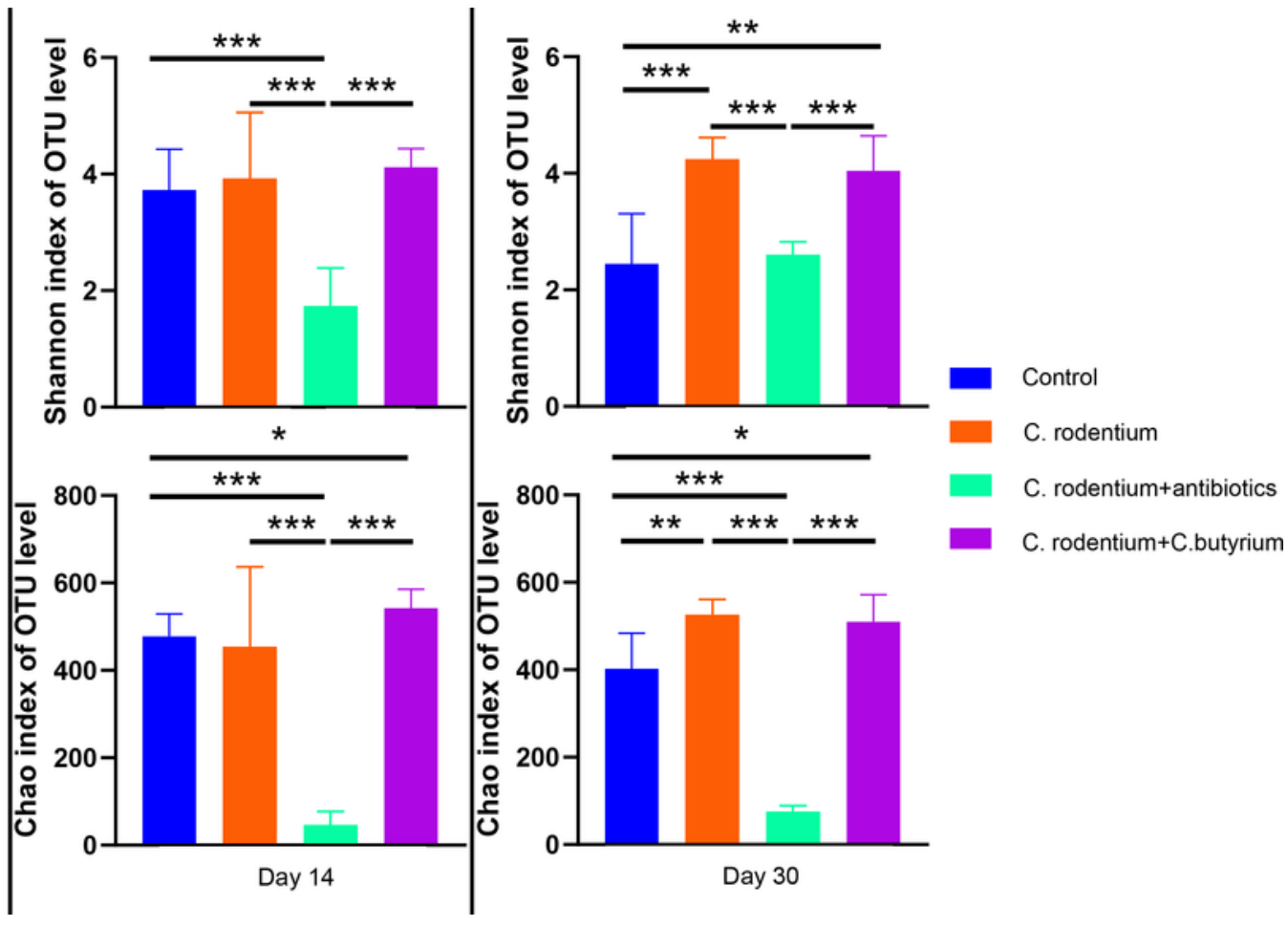


\section{Figure 5}

The $\beta$-diversity of the gut microbiota in the four groups was analyzed based on PCoA at different times.

The structure of the gut microbiota was nearly the same at day $0(R=0.01, P=0.41)$. $C$. rodentium infection did not affect the structure of the gut microbiota compared with the control group $(\mathrm{R}=0.16, P=0.09)$. However, antibiotics treatment significantly affected the structure of the gut microbiota at day $7(R=0.32, P=0.001)$ and day $14(R=0.55, P=0.001)$. After the WAS, the difference between the antibiotic treated group and the other three groups was further increased $(R=0.58, P=0.001)$.
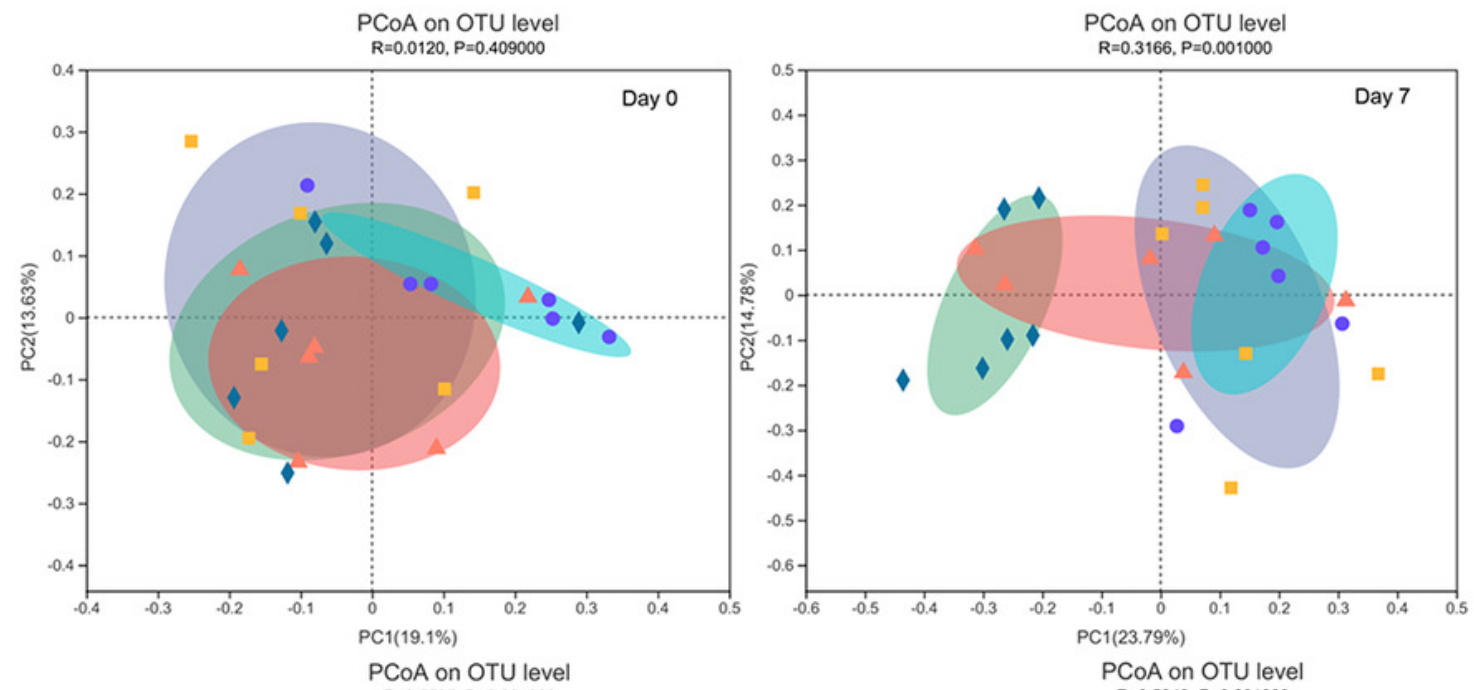

- Control

C. rodentium+antibiotics

C. rodentium $+\mathrm{C}$. butyrium
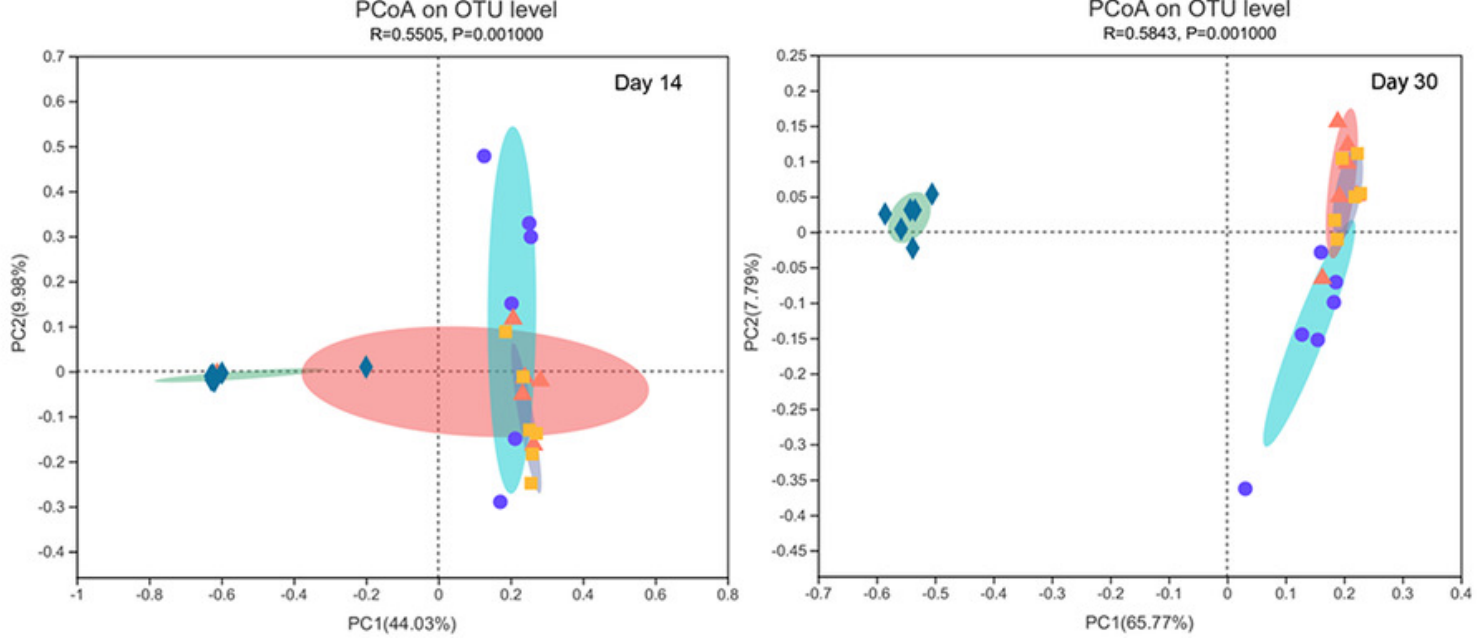


\section{Figure 6}

The microbiota composition changed in different groups.

(A) The main genera of the gut microbiota (the percentages were above 1\%) included more than 38 genera in all groups. (B)The specific taxa that most likely contributed to the differences between the four groups at day 30 were determined by linear discriminant analysis effect size. (C)The amount of genera Lactobacillus and Odoribacter in the four groups were all increased in the $C$. butyricum-treatment group compared with other two C.rodentium infectious groups. The genus Lactobacillus in the control group was higher than those of other groups. $(* P<0.05, * * P<0.01, * * * P<0.001, * * * * P<0.0001)$
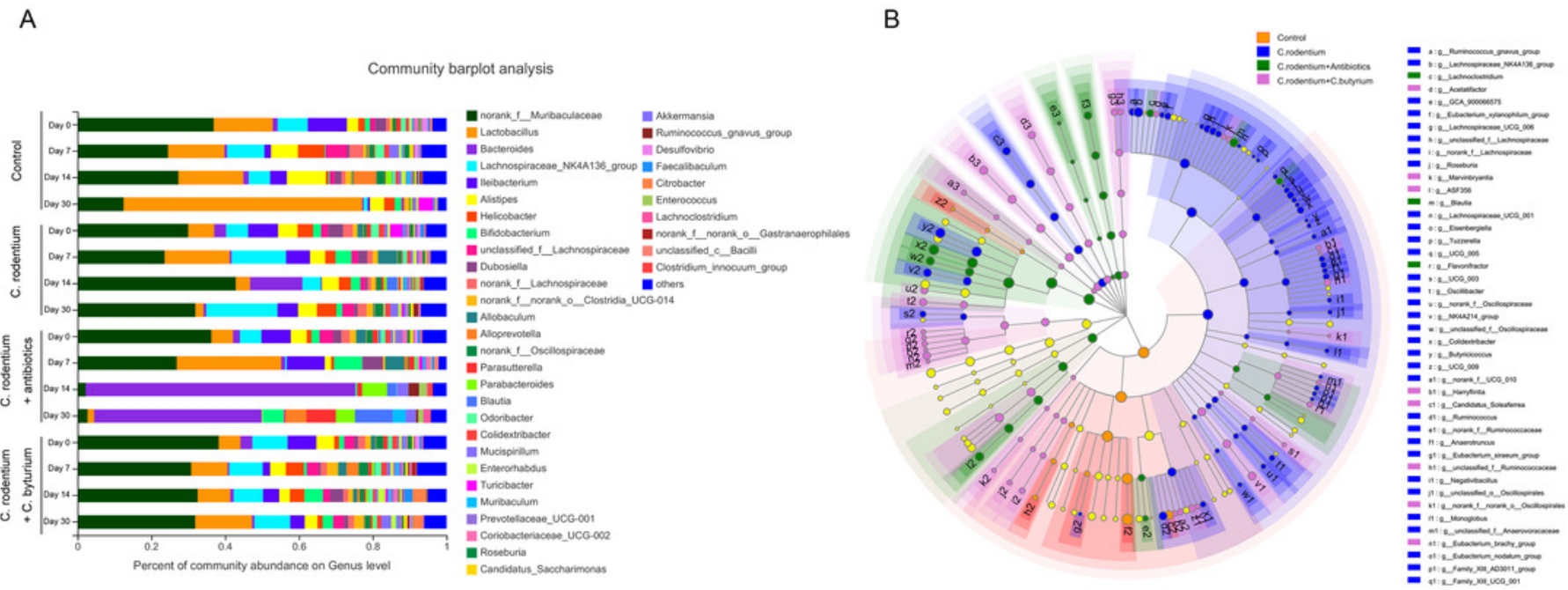

C
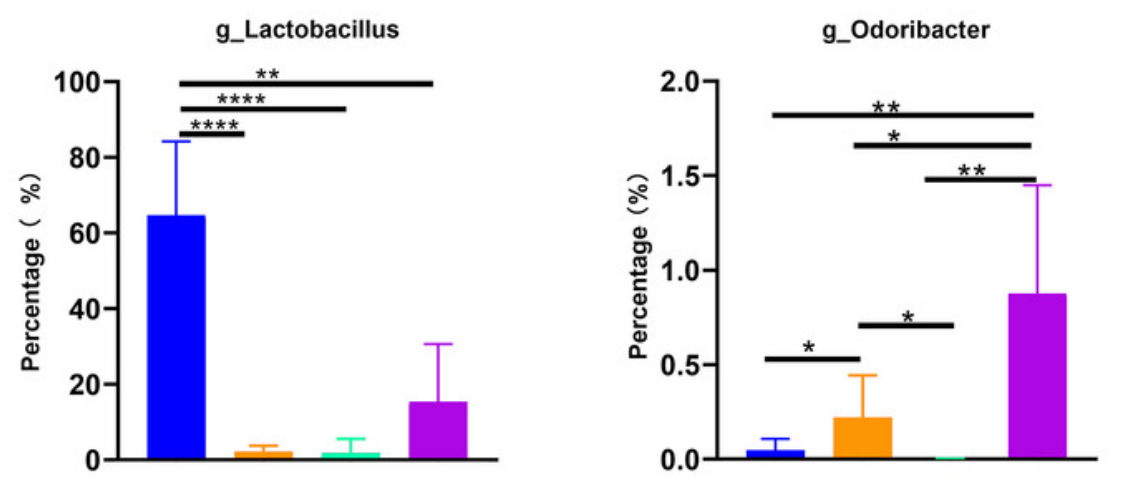

Control

C. rodentium

C. rodentium+antibiotics

C. rodentium + C. butyrium 